\title{
Use of interactive voice response technology to address barriers to fistula care in Nigeria and Uganda
}

\author{
Vandana Tripathi ${ }^{1}$, Elly Arnoff $^{1}$, Benjamin Bellows $^{2}$, Pooja Sripad ${ }^{2}$ \\ ${ }^{1}$ Fistula Care Plus Project, EngenderHealth, Washington, DC, USA; ${ }^{2}$ Population Council, Washington, DC, USA \\ Contributions: (I) Conception and design: All authors; (II) Administrative support: E Arnoff, V Tripathi; (III) Provision of study materials or patients: \\ None; (IV) Collection and assembly of data: E Arnoff, P Sripad; (V) Data analysis and interpretation: E Arnoff, P Sripad, V Tripathi; (VI) Manuscript \\ writing: All authors; (VII) Final approval of manuscript: All authors. \\ Correspondence to: Vandana Tripathi. Fistula Care Plus Project, EngenderHealth, $5059^{\text {th }}$ Street, Suite 601, Washington, DC, USA. \\ Email: vtripathi@engenderhealth.org.
}

Background: The use of digital health technologies has expanded across low-resource settings, including in programs seeking to improve maternal health care seeking and service usage. However, there has been limited use of these technologies for screening and referral within maternal health, and many interventions have relied on SMS tools, which may have limited impact in settings with low female literacy. Digital health technologies have the potential to increase access to care for chronic maternal morbidities, such as obstetric fistula, and for women facing stigma, geographic isolation, and other sociocultural barriers to care seeking. This study documented the process of developing and implementing an innovative fistula screening and referral hotline using interactive voice response (IVR) technology, and described the service usage results and stakeholder perspectives associated with the hotline.

Methods: The IVR hotline was introduced within the context of a broader Fistula Treatment Barriers Reduction Intervention implemented by the USAID-funded Fistula Care Plus project in Ebonyi and Katsina states in Nigeria and Kalungu district in Uganda. The intervention used three communication pathways to disseminate fistula information and conduct fistula screening: trained community agents, trained primary health care providers, and the IVR hotline paired with mass media messaging. All positively-screened women were eligible to receive vouchers for free transportation to an accredited fistula treatment center. Quantitative and qualitative data on intervention implementation and use across all three communication pathways were gathered during intervention implementation, at baseline, midline, and endline; as well as through ongoing program monitoring. This study presents findings specifically on service usage and stakeholder perspectives related to the IVR hotline.

Results: Over a period of ten to twelve months of implementation, depending on the intervention area, a total of 566 women completed the IVR hotline screening process. Across the areas, 415 (73\%) hotline callers screened positive for fistula symptoms. Hotline users and implementation partners reported positive impressions of the hotline, particularly the ability to preserve anonymity in seeking information and referral for fistula symptoms. Challenges to hotline use included limited mobile phone ownership and poor cellular network connectivity, affecting operability by women and community agents.

Conclusions: Implementation of the fistula screening hotline suggests that IVR-based interventions may be useful in expanding access to health services for stigmatized conditions, particularly in settings where literacy is limited. In the current context, such IVR tools require pairing with community and health system partners to complete referral and support clients. Further program experience and evaluation research is required to understand the options for integrating the IVR hotline or other interventions similarly using mobile technologies for screening and referral into broader digital health platforms that are sustained by national health systems or commercial business models.

Keywords: Maternal health; Africa; sub-Saharan; fistula; digital health 
Received: 16 August 2019; Accepted: 18 November 2019; Published: 05 April 2020.

doi: 10.21037/mhealth.2019.12.04

View this article at: http://dx.doi.org/10.21037/mhealth.2019.12.04

\section{Introduction}

\section{Context of digital health platforms for maternal bealth}

Digital health technologies have increasingly expanded in low-resource settings and are recognized as having the potential to improve access to health information and services (1). The role of technology-based health platforms in accelerating progress toward Universal Health Coverage (2) is supported by the 2018 release of the first World Health Organization (WHO) guideline for digital health interventions (3).

Although digital health interventions have been used to promote maternal and child health in many settings, most continue to rely on mobile platforms to "push out" health information or to collect data. A recent review of studies evaluating digital health tools for maternal and newborn health found that health education and promotion was the single most common function, used in nearly half (44\%) of the included papers; and most studies reported use of simple, single function digital health tools that do not align as well with clients' varied needs throughout their life course (4). Digital health interventions are used less frequently to support screening and diagnosis or referral to care (4-8). Most digital health technologies for diagnosis and referral are designed to support health workers, rather than clients or beneficiaries $(5,7)$.

Chronic maternal morbidity is under-recognized and poorly addressed in maternal and child health programs, including those using digital technologies. Programs using such technologies frequently aim to increase antenatal care uptake and facility delivery, and sometimes immediate postpartum/newborn care, but seldom address longerterm post-pregnancy care or design the solution from a continuum of care perspective $(5,7,9)$. This gap is evident in common frameworks that classify and describe digital technologies for health. For instance, the influential Information Communication Technology (ICT) Framework for Reproductive, Maternal, Child, and Adolescent Health (RMNCAH) does not include maternal health content beyond the postnatal period in its array of health topics amenable to ICT intervention (10). However, most chronic disabilities that may be related to pregnancy, such as fistula, pelvic organ prolapse, and anemia, are often not recognized during this immediate phase (11).

Another challenge in realizing the potential of digital health technologies to address maternal health needs is the common reliance on SMS and similar text-based media $(6,7)$. Text-based digital health interventions require functionally literate audiences, and in some low-income populations, a significant percentage of women have low literacy. This may limit the uptake and effectiveness of digital health interventions on the desired health behaviors and outcomes. For example, in Ghana, the Mobile Technology for Community Health Initiative provided pregnant women with information and reminders through its "Mobile Midwife" application. When given the choice between receiving information through voice or SMS messages, $99 \%$ chose voice messages (12). It has been noted that this likely reflects women's relatively lower functional literacy, even in settings such as Ghana where literacy levels are higher than many other sub-Saharan African countries (13). Newer data-rich messaging platforms (e.g., WhatsApp and Telegram) may obviate the either-or proposition of text versus audio by accommodating audio, video, and text in an asynchronous conversational flow.

This context suggests that there are opportunities to expand the ways in which digital health technologies are used to address maternal health needs, including to identify and support women living with chronic pregnancy-related morbidity, and stigmatized conditions, such as female genital fistula.

\section{Fistula and barriers to treatment}

Female genital fistula is a severe morbidity that results in uncontrollable leakage of urine and/or feces from the vagina. It is often obstetric, caused by inadequately managed prolonged or obstructed labor. Fistula can also be iatrogenic, caused by errors during cesarean section or other pelvic surgery procedures. It is estimated that one to two million women around the world live with fistula, primarily in sub-Saharan Africa and South Asia $(14,15)$. Most fistulas can be repaired through surgery or catheter treatment, but many women lack information about and access to fistula services (16). Untreated, fistula can become a devastating chronic disability, leaving women socially isolated, unable 
to work, and frequently abandoned by their spouses or families (17). Comparing estimates of fistula incidence and prevalence to the repair numbers reported to global fistula projects and databases suggests that many women continue to live with untreated fistula even in countries where surgical fistula services have been introduced and are supported by governments and external donors (18-20). Like many conditions that primarily affect the most vulnerable populations, barriers to fistula care are complex. However, these barriers have not been well-understood or examined at the population level. Additionally, there has been very limited evaluation of interventions implemented to increase access to fistula care.

\section{Objectives}

The USAID supported Fistula Care Plus (FC+) project at EngenderHealth and the Population Council collaborated on a research-to-action partnership to understand barriers to fistula care and identify and evaluate interventions to respond to these barriers. The Population Council conducted a global literature review as well as formative qualitative research to understand specific barriers to treatment in Nigeria and Uganda (21-24). Based on the findings of this formative research, the $\mathrm{FC}+$ project designed and implemented the Fistula Treatment Barriers Reduction Intervention in Nigeria and Uganda to improve access to fistula information, screening, and treatment. Paired with transportation vouchers and support from community agents, an interactive voice response (IVR) hotline for fistula screening and referral was a key element of this intervention. The Population Council is conducting an evaluation of the full intervention. The specific aims of this paper are: to describe the process of developing an IVR hotline for fistula screening and referral, contextualized within the broader intervention; to describe the process of implementing this intervention, including synthesizing stakeholder perspectives and documenting modifications to reflect contextual factors; and to report preliminary fistula screening and referral service outputs resulting from the IVR hotline.

\section{Methods}

\section{Theoretical model of barriers to treatment}

The literature review conducted by the Population Council identified a number of barriers faced by women with fistula in acquiring information about and access to fistula diagnosis and treatment services. Baker and colleagues organized and classified these barriers by adapting the three-delays framework frequently used to conceptualize factors contributing to maternal morbidity (21). The resulting theoretical model recognizes that socioeconomic and cultural factors contribute to delays in the decision to seek care; poor accessibility of facilities contributes to delays in the decision to seek care as well as in identifying and reaching a medical facility; and poor quality of care contributes to delays in the decision to seek care as well as in receiving adequate and appropriate treatment once a facility is reached. This literature review also identified 23 articles describing interventions to address barriers to fistula care. However, documentation and evaluation of these interventions was limited, resulting in little ability to draw inferences about their contributions to improved treatment access or generalizability to other settings (21).

Following the literature review, formative research was conducted in Nigeria and Uganda to elucidate specific barriers to and enablers of fistula care in these settings (22-24). The formative research consisted of in-depth interviews with fistula patients, their family members, health service providers, and health systems managers; and focus group discussions with women who had received fistula repair and male and female community stakeholders. The formative research data collected was conducted in Kano and Ebonyi states in Nigeria, and Hoima and Masaka districts in Uganda.

The literature-based theoretical model and these context-specific formative research findings informed the development of the Fistula Treatment Barriers Reduction Intervention. Findings from the formative research have been presented extensively elsewhere (22-24). However, four notable barriers identified through formative research suggested a role for an IVR-based digital health solution in improving access to fistula treatment. First, the research indicated a persistent impact of stigma. In some settings, women with fistula face such shame and isolation due to their symptoms that they do not participate in any community events. Therefore, in-person community education, outreach, and mobilization activities designed to share information about fistula and other maternal health issues may be failing to reach them $(23,24)$. Second, the research suggested that 'gatekeeping' by primary health center (PHC) providers may create hurdles that prevent women from reaching appropriate service points for fistula 


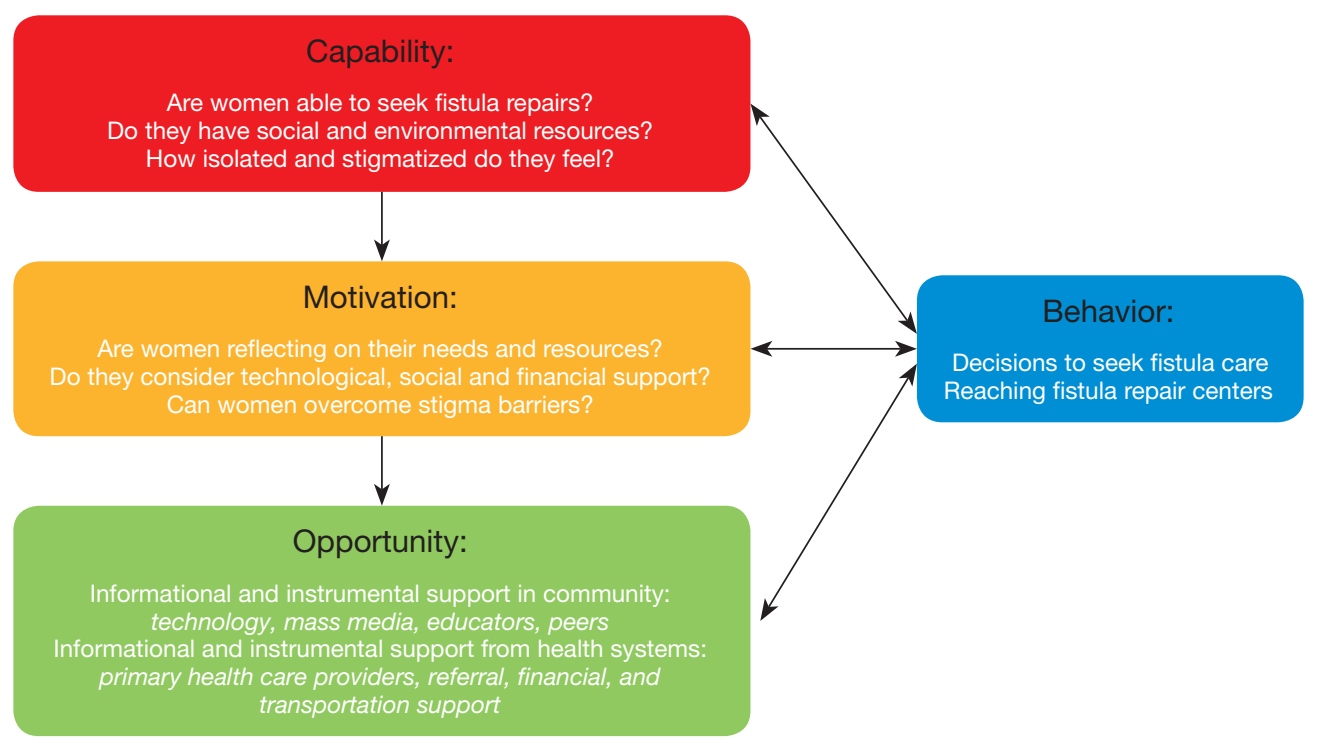

Figure 1 The COM-B Model for fistula care seeking. COM-B, Capability, Opportunity, and Motivation determines Behavior.

diagnosis and treatment $(23,24)$. PHC providers are not well-informed about fistula, its symptoms and treatment, or the location of fistula centers that are equipped to provide diagnostic clinical examinations and surgical or nonsurgical fistula repair. Therefore, women frequently receive inaccurate information or even unskilled attempts at fistula treatment at PHC sites, rather than being appropriately referred onward to fistula centers $(23,24)$. Third, many women live at a great distance from these fistula centersthe financial transportation and opportunity costs of reaching these services prevent them from seeking and reaching appropriate diagnosis and treatment (22-24). Finally, in low-resource settings, many women with fistula have low or no literacy; this was observed in our formative research settings and has been reported in other studies of this population (23-25). Therefore, fistula communication messages transmitted through SMS or other written formats will be not be effective in reaching them.

\section{Fistula treatment barriers reduction intervention: role of IVR hotline}

The Fistula Treatment Barriers Reduction Intervention sought to address the barriers described above, focusing on those that could be addressed effectively in the context of a time-bound project and existing local partnerships. The intervention design was guided by the Capability, Opportunity, and Motivation determines Behavior
(COM-B) Model, synthesized from a review of 19 behavior change frameworks by Michie and colleagues and applied in a variety of health service research settings (26-28). The COM-B Model, adapted for the context of fistula care seeking, is illustrated in Figure 1.

The FC+ intervention was designed to increase women's capability, motivation, and opportunity to decide to seek and to access fistula screening and treatment services. To achieve this, the intervention used three communication pathways to disseminate fistula information and screening resources: trained community agents, trained PHC providers, and a free IVR hotline paired with mass media messaging. Written job aids were produced for community agents and PHC providers to take women through the screening algorithm, mirroring the script of the IVR hotline. Each pathway provided consistent, accurate information on fistula symptoms and treatment, including a single, streamlined fistula screening algorithm. Three pathways were used because the formative research revealed that different barriers may affect different women; the presence of multiple, consistent pathways across settings was intended to improve information and access for all women living with fistula, whatever their specific cluster of experienced barriers.

Positively-screened women were eligible for the same enabler through each pathway-a voucher for transportation for her and a companion to travel to and from an accredited fistula treatment center, where she could 


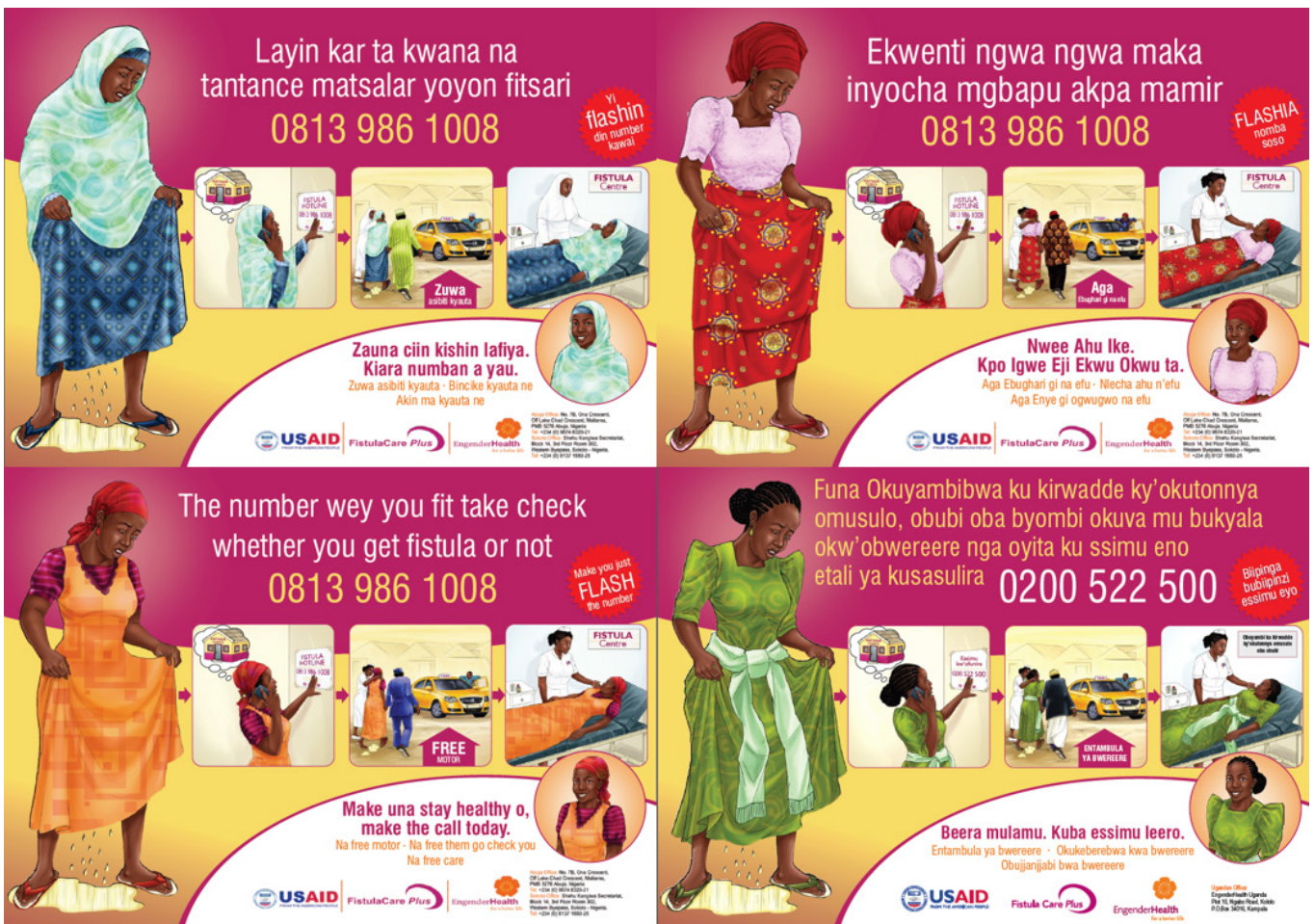

Figure 2 Communication materials advertising the IVR hotline in Nigeria and Uganda in the Hausa, Igbo, Nigerian Pidgin, and Luganda languages. IVR, interactive voice response.

receive clinical diagnosis and treatment, as appropriate. The full intervention has been described elsewhere (29) and the description here focuses on the IVR hotline.

FC+ collaborated with Viamo (formed in 2017 from a merger of VOTO Mobile and Human Network International), a company that uses a digital-over-mobile platform for data collection and the provision of public service information to consumers via SMS and IVR. The $\mathrm{FC}+$ hotline used pre-recorded messages by voice actors to take callers through a process of screening for fistula symptoms, collection of background information, and provision of action messages depending on the screening result. At the start of the call, women were able to select their preferred language. Callers were asked their age, followed by a key fistula screening symptom question: " $D$ o you currently experience constant leakage of urine or feces from your vagina during the day and night even when you are not urinating or trying to urinate?" Callers who responded "yes" were asked questions about their demographic background, perceived cause of fistula, and barriers they have experienced in seeking treatment. Women who were positively screened and lived within the intervention areas were told that they would receive follow up from a community agent within four days. Women who were positively screened but lived outside the intervention area received follow-up messages, both voice and SMS, with information about the nearest fistula treatment center. In order to reduce cost-related barriers to care, the hotline was designed so that callers "flash" into a toll-free number and receive a prompt call back.

A prototype version of the IVR hotline was tested for issues including clarity of terms and acceptability of voices, and the script and audio recordings were revised to reflect user feedback. In addition to mass media advertisements, the fistula hotline was widely advertised through graphic, low-text flyers disseminated by community agents and PHC providers at community venues (see Figure 2).

Figure 3 illustrates the role of the IVR hotline in the fistula screening and referral process, in both Uganda and Nigeria.

\section{Setting}

FC+ implemented the Fistula Treatment Barriers Reduction intervention in Nigeria and Uganda, both of which have a well-documented fistula burden. According to the 


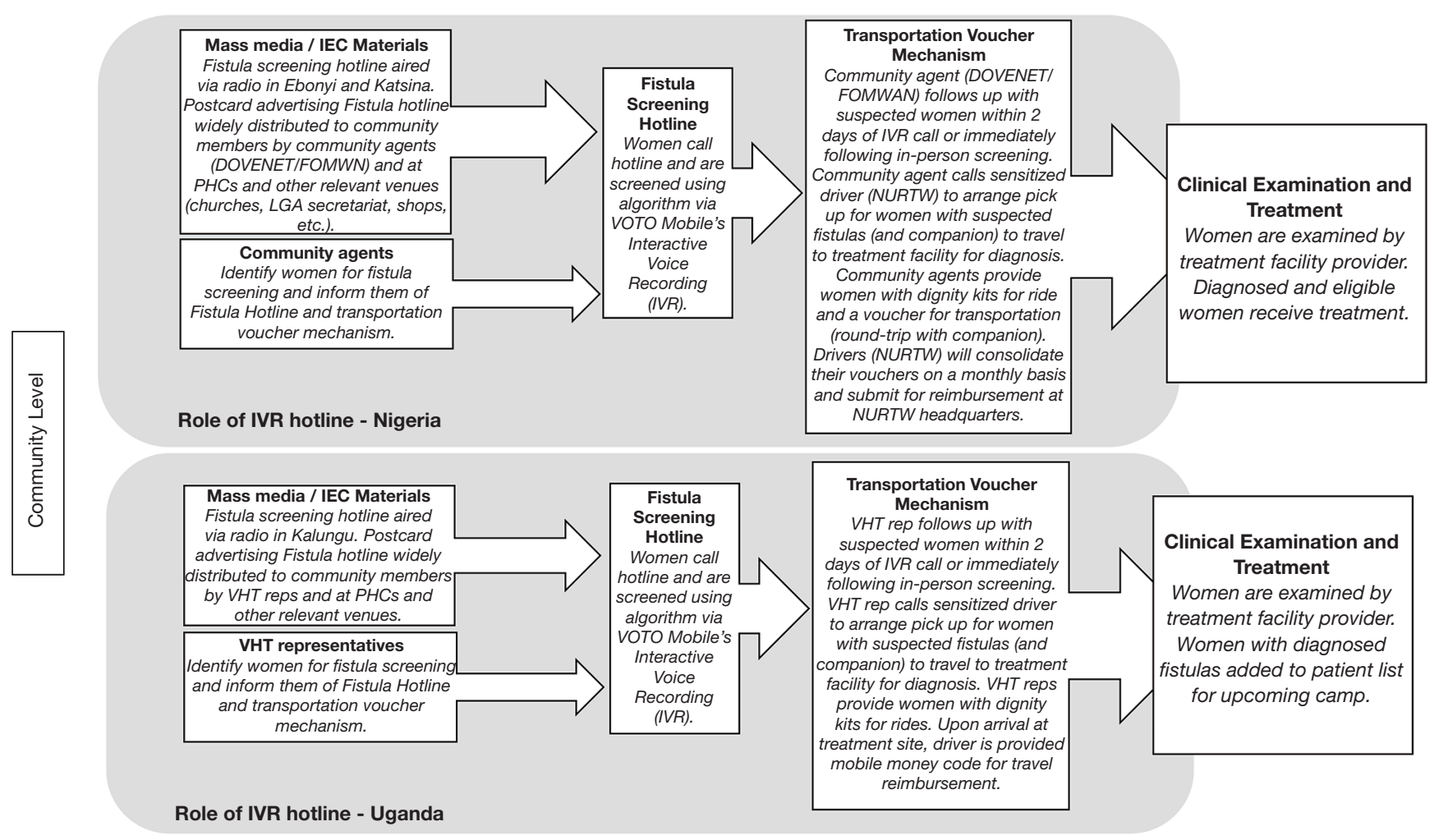

Figure 3 Role of IVR hotline in fistula screening and referral in Nigeria and Uganda. IEC, information, education and communication; IVR, interactive voice response.

Demographic and Health Surveys, $0.4 \%$ of women of reproductive age in Nigeria report ever experiencing fistula symptoms and $1.6 \%$ of women in Uganda report lifetime experience of such symptoms (30,31). Applying correction factors, Maheu-Giroux and colleagues have estimated that this translates to 46,800 women currently living with fistula in Nigeria and 74,200 in Uganda (32). Both countries have implemented national and local efforts to establish fistula treatment centers, including in collaboration with international partners.

The FC+ project implemented the Fistula Treatment Barriers Reduction Intervention in in two areas within Nigeria: Ikwo local governance area (LGA) in Ebonyi state, and Katsina LGA in Katsina state; and one area within Uganda: Kalungu district. An accredited fistula treatment center served as the key service delivery point for each intervention area, to which all positively-screened women were referred through the intervention. In Nigeria, women were referred to the National Obstetric Fistula Center (NOFIC), Abakaliki, in Ebonyi and the NOFIC, Babbar Ruga, in Katsina. In Uganda, women from Kalungu district were referred to the Fistula Clinic at Kitovu Mission Hospital in Masaka district.

In Nigeria, community agents participating in the intervention worked for two community-based organizations, Daughters of Virtue and Empowerment (DOVENET) in Ebonyi and the Federation of Muslim Women in Nigeria (FOWAM) in Katsina. In Uganda, community agents were members of Village Health Teams (VHTs), which are groups of community health worker volunteers formally affiliated with the Ministry of Health.

To reflect languages spoken in these settings, the IVR hotline's pre-recorded audio was available in four languages: Hausa, Igbo, and Nigerian Pidgin in Nigeria; and Luganda in Uganda.

While the intention was to restrict all three interventions screening and referral pathways to the intervention areas, in Nigeria this proved challenging for the IVR hotline. Because the mass media advertisement of the hotline had to be delivered through state radio, it was not possible to prevent callers from outside the intervention area from receiving this information and calling the hotline. As 
Table 1 Data collection to evaluate the fistula treatment barriers reduction intervention

\begin{tabular}{|c|c|c|c|c|c|c|c|c|c|}
\hline \multirow{2}{*}{ Data source } & \multicolumn{3}{|c|}{ Baseline $^{\dagger}$} & \multicolumn{3}{|c|}{ Midline } & \multicolumn{3}{|c|}{ Endline } \\
\hline & Ebonyi & Katsina & $\begin{array}{c}\text { Uganda } \\
\text { Central } 1 \\
\text { Sub-Region }\end{array}$ & Ebonyi & Katsina & 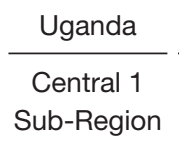 & Ebonyi & Katsina & $\begin{array}{c}\text { Uganda } \\
\text { Central } 1 \\
\text { Sub-Region }\end{array}$ \\
\hline Facility assessments of PHCs & 39 & 37 & 50 & $\mathrm{~N} / \mathrm{A}$ & N/A & N/A & 38 & 31 & 43 \\
\hline Surveys of post-repair women & 91 & 81 & 96 & N/A & N/A & N/A & 51 & 44 & 47 \\
\hline $\begin{array}{l}\text { In-depth Interviews with critical stakeholders: } \\
\text { beneficiaries and implementers }\end{array}$ & $30^{\star}$ & $30^{*}$ & $29^{*}$ & 19 & 18 & 18 & 11 & 19 & 18 \\
\hline $\begin{array}{l}\text { Focus group discussions with men and women } \\
\text { residing in selected communities }\end{array}$ & 0 & 4 & 6 & $\mathrm{~N} / \mathrm{A}$ & $\mathrm{N} / \mathrm{A}$ & $\mathrm{N} / \mathrm{A}$ & 8 & 8 & 8 \\
\hline Dissemination and feedback meetings & \multicolumn{3}{|c|}{ N/A } & \multicolumn{3}{|c|}{3} & \multicolumn{2}{|l|}{3} & \\
\hline
\end{tabular}

a result, hotline usage in Nigeria includes callers from Ebonyi and Katsina States as a whole, rather than only the intervention areas.

The intervention was implemented from June 2017 to April 2018 in Ebonyi, July 2017 to April 2018 in Katsina, and July 2017 to June 2018 in Kalungu.

\section{Data collection}

Table 1 summarizes data collection for the evaluation of the overall Fistula Treatment Barriers Reduction Intervention.

Process and output data to evaluate the comprehensive intervention were gathered through multiple qualitative and quantitative sources, including PHC assessments; fistula center record reviews; surveys of $\mathrm{PHC}$ providers and women who had received fistula repairs; and interviews and focus group discussions with stakeholders, including health providers, fistula clients, community agents, transportation partners, and health system managers. Quantitative data collection documented health facility capacity, provider and stakeholder knowledge and practices, and service usage and outputs at health facilities as well as through the three intervention pathways. Qualitative data collection explored stakeholders' experiences of the implementation process, including ways in which the intervention was modified over the course of the implementation period to respond to contextual realities. Qualitative data collection also examined fistula clients' and community members' perspectives on barriers and enablers to accessing fistula care and normative attitudes toward fistula causes and consequences.

Baseline evaluation data were collected in all three sites shortly before the intervention was initiated; midline assessments were conducted between four and five months into intervention implementation; and endline evaluation data were collected in each site after the implementation period was complete. Feedback meetings to disseminate learnings from the intervention were conducted in all three sites, and used as additional opportunities to collect stakeholder perspectives.

Monitoring data, including monthly services statistics at fistula treatment centers, were compiled on an ongoing basis by intervention staff. A facility monitoring tool was provided to intervention area fistula treatment centers to enable ongoing documentation of fistula clients' exposure to the IVR hotline and other intervention pathways, as well as experienced barriers to fistula care. Monitoring data collection unique to the IVR hotline included ongoing review of call logs and meta-data from the Viamo platform.

For this specific study of the IVR hotline, quantitative summaries of hotline use and referral outputs were produced across all three intervention areas. Qualitative data were analyzed by a team that jointly developed a codebook of emergent themes based on in-depth reading of the transcripts. Researchers used NVivo 11 software 
Table 2 IVR fistula hotline usage 2017-2018

\begin{tabular}{lcc}
\hline Output & Ebonyi & Katsina \\
\hline Number of unique callers completing IVR hotline screening ${ }^{\dagger}$ & 301 & 144 \\
Number (percent) of callers screened positively for fistula & $228(76 \%)$ & $101(70 \%)$ \\
Number of positive-screened women referred to and receiving & $118(18$ lkwo LGA) & 99 (9 Katsina LGA) \\
diagnosis at facility & 41 \\
\hline ', this includes callers who responded to the primary fistula symptom screening question and were not calling from a phone number that \\
had already called the hotline within a short time frame. This figure excludes callers from outside Kalungu district in Uganda. It includes \\
callers from within Ebonyi state and Katsina state but outside the Ikwo and Katsina LGAs. See the Methods section for the rationale for \\
including callers from outside the intervention areas in Nigeria. IVR, interactive voice response; LGA, local governance area.
\end{tabular}

to organize and code transcripts, from which charts and narrative summaries were generated. This manuscript draws on narrative summaries and quotations from interviews related to implementation and experience of the IVR hotline.

\section{Ethical considerations}

In the United States, the research protocol for the evaluation of the Fistula Treatment Barriers Reduction intervention was reviewed and approved by the Institutional Review Board (IRB) at the Population Council in New York. In Nigeria, the protocol was also reviewed by the National Health Research Ethics Committee at the Federal Ministry of Health (FMOH) and the Ebonyi and Kano State Health Research Ethics Committees. In Uganda, the protocol was reviewed by the School of Medicine Research and Ethics Committee at Makerere University and the Uganda National Council for Science and Technology.

\section{Results}

\section{Service outputs}

Table 2 provides outputs related to IVR hotline implementation in 2017 and 2018, synthesized from ongoing monitoring of the Viamo platform and facility monitoring tools. Between June 2017 and April 2018, 301 unique callers contacted the IVR hotline and completed the key screening questions in Ebonyi. Of these, 228 (76\%) were screened positively based on their response to the primary fistula symptoms question. Between July 2017 and April 2018, 144 unique callers were screened via IVR in Katsina, with 101 (70\%) screening positive. Between July 2017 and June 2018 in Kalungu. One hundred and twentyone unique callers were screened via IVR in Kalungu, with $86(71 \%)$ screening positive. In Nigeria, 118 women in Ebonyi and 99 women in Katsina referred through the intervention received diagnosis services at the fistula treatment center; and in Uganda, 41 referred women from Kalungu received diagnosis services.

Figure 4 illustrates quarterly hotline usage in the two sites in Nigeria during the quarters when the hotline was active in both Katsina and Ebonyi. This demonstrates that calls to the hotline peaked in the second and third quarters of hotline availability. This increase was driven primarily by increased calls in Katsina in these quarters.

\section{Intervention modifications during implementation}

Data from ongoing monitoring as well as qualitative data collection at midline and endline revealed the ways in which the Fistula Treatment Barriers Reduction Intervention was modified from the initial program plan during implementation. The amendments reflected responses to challenges identified by implementation stakeholders.

A number of modifications addressed the function of the IVR hotline. Early in the implementation period, it became evident that many community agents themselves had low or no literacy and were themselves using the IVR hotline to screen and refer women. The training and guidance for intervention partners were revised to explicitly incorporate the hotline and describe a process for IVR-facilitated screening by community agents. Poor cellular connectivity created challenges in the efficient use of the hotline and required partners to adapt their approaches. For example, in one Uganda locality, connectivity was stronger at night, so VHT representatives shifted to calling the fistula program coordinator later at night. Community agents also identified hot spots for cellular connectivity in their villages and disseminated information about these among their peers, to 


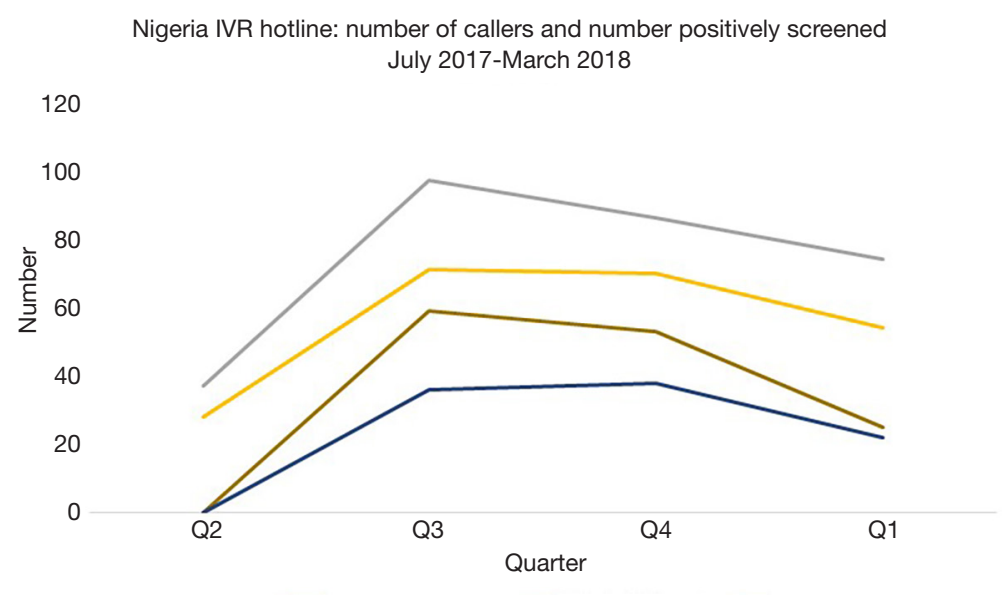

Figure 4 Quarterly hotline usage and positive screening.

improve their ability to follow-up with positively-screened women.

Women's limited ownership of phones and the frequent use of a single phone by many individuals threatened confidentiality during the follow-up call process. To respond to this, the program developed a protocol for community agent call-backs to promote confidentiality and ensure that screening results were only discussed with the woman who had called the hotline, and not friends or other household members.

Community partners also felt that, despite mass media advertisement and communication materials, awareness of the IVR hotline was inadequate. Larger-format posters were produced and displayed at PHC facilities as well as distributed through community agents, to improve knowledge about the hotline. Providing refresher training and ensuring adequate monitoring of hotline calls and coordination among the community agents providing referral and transportation support were perceived as necessary to effective functioning of the IVR hotline by the implementation partners.

Documentation of the implementation process also revealed variations in implementation due to site-specific needs and preferences. In Ebonyi, language comprehension posed a challenge despite pre-testing of messages, necessitating adequate retranslation of the IVR platform. In Katsina and Kalungu, comparison of facility records with intervention monitoring data indicated that some women preferred to travel to the fistula treatment center independently, without using the transportation voucher. Additionally in Kalungu, intervention staff reported preferring to take women who were screened positively through the hotline to a PHC center for an initial followup, before traveling to the fistula treatment center. This may have resulted in some women being diagnosed with other non-fistula conditions at the PHC level, thereby further reducing the proportion of positively-screened women who used the transport voucher or arrived at the fistula treatment facility in Uganda.

\section{Stakebolder perspectives}

During midline and endline interviews and focus group discussions (33-35), most stakeholders reported that the IVR hotline was a helpful tool in broadly disseminating fistula treatment information. Community agents found the hotline to be helpful in organizing their fistula screening and referral process.

"With that hotline, they give you directions properly and you don't lose track. It helps women because it gives them directives... there is a health worker who responds to you and tells you to go to Kitovu like this and that."-Community agent, Uganda

Respondents noted that the hotline helped reduce stigma associated with disclosing fistula symptoms, with some community agents attributing client volume at fistula treatment centers to the hotline.

"Because of stigma, the person thinks, 'it's just me and the radio gives the number' - (she) calls the number... it was a wonderful strategy and really helped."-Health program manager, Ebonyi, Nigeria

"I would tell them that if they hear of anyone with the problem, utilize that number... (if she) does not want to tell you 
her secret, she can dial it privately."-Community agent, Uganda

"When you have the number, you will not even see the women... for instance, in a month you will not have (referred) anybody that has the problem, but they will be plenty in the hospital due to the hotline number. They will just go directly." Community agent, Katsina, Nigeria

Across sites, the complementarity of community agents and the hotline was critical for effective referral and transport to the fistula center. Respondents reported that, to be effective, the hotline requires enough publicity through radio, peer or PHC promotion.

"There is a VHT who came and told me, 'there are some flyers which have some numbers which you can come and get and try to call them.' I went and she gave me that number and I called them."-Fistula client, Uganda

"At times even if it's not the number, they listen to the announcements over the radio and they come asking me about it that, 'We heard an announcement over the radio, is it true?' Then I tell them that is true, that is how it is, and tell them to go there."-Community agent, Uganda

Respondents identified challenges in using the IVR hotline as intended. In Uganda, community agents faced difficulty in linking with hotline callers for follow up and community skepticism about the confidentiality afforded by community-agent-initiated calls. Across sites, some respondents were confused about how to use the hotline and reported challenges related to poor network coverage and connectivity in the intervention areas.

"The network in our villages is a problem, so one might call the hotline and the network fails which becomes a problem... one patient has been calling now for a week; the number is not available."-Driver, Uganda

"Using the botline is (difficult); not everybody has a phone and not everybody knows how to operate a phone except by using the community volunteer" - Community agent, Katsina, Nigeria

Additionally, some women who successfully used the hotline for screening and accessed fistula treatment facilities complained about speaking to a recording, rather than a real person.

"I was about to lose hope. I wondered why we cannot get to people and instead only the computer voices."-Fistula client, Uganda

During endline dissemination meetings to share learning and solicit feedback from the Fistula Treatment Barriers Reduction Intervention, local and national stakeholders reflected on the hotline. They expressed enthusiasm for wider community education about fistula, to shift potentially stigmatizing norms and empower women with fistula to seek care. In Katsina, media outlets interested in corporate social responsibility considered sustaining fistula awareness campaigns to relay care options through television and radio. In Ebonyi, stakeholders advocated for the placement of a desk officer at the local government level to connect women to fistula treatment facilities. In Uganda, mechanisms for building awareness were prioritized in discussions of the National Fistula Strategy. Despite the wide reach and promise of the hotline across sites, there was a collective recognition of the need for national support and financial commitment to sustain such mechanisms, particularly through integration with routine referral systems.

\section{Discussion}

The IVR hotline was widely used in intervention areas in Nigeria and Uganda to identify and refer women with fistula symptoms, both by women themselves and through facilitated calls with community agents.

\section{Strengths}

This paper describes a novel application of a client-facing IVR platform to support fistula screening and referral, and indicates its utility in increasing awareness and use of fistula services by women in Nigeria and Uganda. Documentation of the implementation experience through qualitative and quantitative data from multiple sources enabled an in-depth examination of how digital health technologies can be used for client-directed screening and referral in settings of limited health service access, and how IVR tools can address barriers inherent in text-based digital health technologies.

\section{Limitations}

The hotline was implemented as a pilot for up to one year, depending on the site, in relatively limited geographic areas. At this time, there is not funding to continue the hotline through local resources and the hotline is not currently active. This precluded a robust assessment of sustainability. Future work could consider strategies to strengthen sustainability including disseminating the content through a commercial digital health platform or recruiting local partners to take on hotline maintenance and conduct consistent follow-up for positively-screened women $(36,37)$. Additionally, during this study, it was not possible to assess population exposure to mass media and other 
advertisement of the hotline, to gauge the potential for expanded impact with additional saturation of population awareness of the hotline. Community partners' feedback during implementation indicated limited awareness of the hotline, suggesting that the ability of the intervention to reach those women most isolated due to stigma could be improved. In Uganda, recent research indicates that about $58.5 \%$ of women listen to a radio at least once a week; given this usage rate, mass media advertisements may have to be quite frequent to achieve greater awareness of digital health interventions such as the hotline (31).

\section{Potential population impact}

Applying the service outputs documented through this preliminary analysis, it is possible to estimate the impact of this screening and referral intervention on the populationlevel fistula burden.

Not all women who are positively screened will be positively diagnosed with fistula upon clinical examination. Monitoring data does not allow us to determine which of the positively screened women were confirmed to have fistula upon diagnostic examination at the fistula treatment center. However, a study in Nigeria pairing a similar symptom-based fistula screening question with clinical diagnostic examination of positively-screened women found the screening to have a $47 \%$ positive predictive value (PPV) (38). Applying this PPV to the number of positively screened callers, it can be estimated that approximately 107 fistula cases were identified in Ebonyi, 47 in Katsina, and 40 in Kalungu District. It is likely that most positivelyscreened women who do not have fistula do have severe incontinence from other causes, which can also potentially be diagnosed and addressed once they are seen at a fistula treatment center.

These figures can be compared to estimates of fistula prevalence in Uganda and Nigeria in order to provide a preliminary assessment of the proportion of women living with fistula identified through the IVR hotline. As noted above, researchers have applied correction factors to Demographic and Health Survey data on self-reported lifetime experience of fistula symptoms to estimate current fistula prevalence in sub-Saharan African countries (32). They estimated that 46,800 women are living with fistula in Nigeria, and 74,200 in Uganda. Fistula is not evenly distributed within countries-factors such as population density, access to emergency obstetric care, and fertility rates can affect fistula prevalence in specific regions and states.
However, assuming proportional geographic distribution of fistula and applying the most recent available population figures (39-41), it can be estimated that 696 women are living with fistula in Ebonyi state (69 in the Ikwo LGA) 1,895 women in Katsina state (104 in Katsina LGA), and 758 women in Kalungu district. Based on these assumptions, approximately $15.4 \%$ of fistula cases in Ebonyi State, $2.5 \%$ in Katsina State, and $5.3 \%$ in Kalungu District were screened through a nine to twelve-month implementation of the IVR hotline. The Ebonyi and Katsina proportions are more conservative as they reflect the populations of the states rather than the intervention areas.

\section{Program and research implications}

The use of a client-facing IVR interface integrated with complementary health systems and community support appears promising to improve access to care for women living with fistula, particularly those who face care-seeking barriers such as stigma, low literacy, and social or geographic isolation. The hotline may also help women avoid delays in care seeking due to inadequate capacity at PHC sites, as it appears user-friendly and effective in reaching large populations, if accompanied by adequate publicity to community health partners. While the estimated proportion of fistula cases identified through hotline screening is relatively low $(2.5-15.4 \%)$, this may reflect the limited geography and short duration of implementation within the study context. The impact may be significantly greater if the IVR screening approach can be implemented for a longer period of time, advertised more broadly, and integrated with other population-oriented digital health platforms, whether these are managed by national health systems or through commercial business models.

User feedback and stakeholder perspectives indicate challenges in efficient and effective hotline use. Language comprehension requires careful consideration and may pose challenges to scale up in linguistically diverse settings. Variable mobile connectivity also currents limits scale up in the very areas where poor access to care increases fistula risk.

Notably, while the intervention sought to address barriers that are magnified by gender inequity (e.g., women's limited control of financial resources, relatively lower literacy, and vulnerability to provider gatekeeping), the implementation process uncovered efficiency and effectiveness challenges driven by the same gender issues. For instance, our experience concurred with other research 
in Uganda and Nigeria, as well as sub-Saharan Africa more broadly, indicating that women are less likely to own their own phones and more likely to share a single phone with multiple other family members $(31,42,43)$. This presented challenges to confidentiality, made the process of follow-up for referral more cumbersome, and may have reduced some women's willingness to make use of transportation vouchers.

Crucially, the necessity of in-person contacts for referral follow-up and transportation support suggest that a digital health intervention alone may not yield sufficient impact in addressing barriers to fistula treatment in low-resource settings. This may change as phone ownership expands and tools, such as mobile money, are more widely accessible and used in lower-resource settings.

While most examinations of digital health solutions for maternal health have focused on text-based applications, our experience aligns with certain lessons identified in these reviews. Ahmed and colleagues identified success factors, including technology (e.g., network coverage, technical support), factors promoting user acceptance, funding, organization factors (e.g., effective coordination), and political support (e.g., government support to scale technologies) (44). Consideration of such factors and learning from this implementation experience provide guidance for how IVR-based screening and referral for fistula and other similarly challenging health conditions can be refined and expanded in future iterations. Should this IVR intervention be delivered at an expanded scale, it will be important to monitor population exposure and the process of implementation, to identify, as Lefevre and colleagues have emphasized, who the intervention is reaching, what dosage the intended target population is receiving, and where "critical breaks" in the delivery of the intervention and follow-up services occur (45).

The monitoring and evaluation of this implementation experience has appropriately provided information relevant at the early stages of a digital health intervention (46), such as functionality, usability, efficacy, and feasibility. Future research on the role of the IVR hotline in addressing barriers to fistula treatment can examine more complex, population-oriented results, including quality and costeffectiveness.

It is important to note that the IVR intervention was nested within a comprehensive intervention providing other pathways for fistula screening and referral. This paper only presents preliminary results from analysis of quantitative and qualitative data collected at the endpoint of the overall intervention. The final evaluation of the Fistula Treatment
Barriers Reduction Intervention's effects is currently being completed by the Population Council. Findings regarding the total influence of the Fistula Treatment Barriers Reduction intervention on fistula case detection, barriers to, and completion of treatment will be reported separately when this evaluation is complete.

\section{Conclusions}

This experience implementing the fistula screening hotline suggests that IVR-based interventions may be particularly useful in settings where literacy is limited to scale access to health services for stigmatized conditions. However, such interventions ought to be linked to complementary referral and support systems to optimize impacts for community members. Further program experience and research is required to understand the options for integrating the IVR hotline, or similar interventions using mobile technologies, for screening and referral into broader digital health platforms that are sustained by national health systems.

\section{Acknowledgments}

The authors thank Erin Mielke and Mary Ellen Stanton of the United States Agency for International Development (USAID) for the review of this manuscript. They also thank Charity Ndwiga of Population Council-Kenya for her support in qualitative data collection and interpretation. This study is made possible by the support of the American People through USAID.

Funding: None.

\section{Footnote}

Conflicts of Interest: The authors have no conflicts of interest to declare.

Ethical Statement: The authors are accountable for all aspects of the work in ensuring that questions related to the accuracy or integrity of any part of the work are appropriately investigated and resolved. In the United States, the research protocol for the evaluation of the Fistula Treatment Barriers Reduction intervention was reviewed and approved by the Institutional Review Board (IRB) at the Population Council in New York. In Nigeria, the protocol was also reviewed by the National Health Research Ethics Committee at the Federal Ministry of Health (FMOH) and the Ebonyi and Kano State Health Research Ethics 
Committees. In Uganda, the protocol was reviewed by the School of Medicine Research and Ethics Committee at Makerere University and the Uganda National Council for Science and Technology.

Open Access Statement: This is an Open Access article distributed in accordance with the Creative Commons Attribution-NonCommercial-NoDerivs 4.0 International License (CC BY-NC-ND 4.0), which permits the noncommercial replication and distribution of the article with the strict proviso that no changes or edits are made and the original work is properly cited (including links to both the formal publication through the relevant DOI and the license). See: https://creativecommons.org/licenses/by-ncnd/4.0/.

\section{References}

1. Tran Ngoc C, Bigirimana N, Muneene D, et al. Conclusions of the digital health hub of the Transform Africa Summit (2018): strong government leadership and public-private-partnerships are key prerequisites for sustainable scale up of digital health in Africa. BMC Proc 2018;12:17.

2. United Nations. Resolution A/RES/70/1. Transforming our world: the 2030 agenda for sustainable development. In: Seventieth United Nations General Assembly, New York, 25 September 2015.

3. WHO guideline: recommendations on digital interventions for health system strengthening. World Health Organization, Geneva, 2019.

4. Chen H, Chai Y, Dong L, et al. Effectiveness and Appropriateness of mHealth Interventions for Maternal and Child Health: Systematic Review. JMIR Mhealth Uhealth 2018;6:e7.

5. Feroz A, Perveen S, Aftab W. Role of mHealth applications for improving antenatal and postnatal care in low and middle income countries: a systematic review. BMC Health Serv Res 2017;17:704.

6. Lee SH, Nurmatov UB, Nwaru BI, et al. Effectiveness of mHealth interventions for maternal, newborn and child health in low- and middle-income countries: Systematic review and meta-analysis. J Glob Health 2016;6:010401.

7. Colaci D, Chaudhri S, Vasan A. mHealth Interventions in Low-Income Countries to Address Maternal Health: A Systematic Review. Ann Glob Health 2016;82:922-35.

8. Watterson JL, Walsh J, Madeka I. Using mHealth to Improve Usage of Antenatal Care, Postnatal Care, and
Immunization: A Systematic Review of the Literature. Biomed Res Int 2015;2015:153402.

9. Sondaal SF, Browne JL, Amoakoh-Coleman M, et al. Assessing the Effect of mHealth Interventions in Improving Maternal and Neonatal Care in Low- and Middle-Income Countries: A Systematic Review. PLoS One 2016;11:e0154664.

10. Labrique AB, Vasudevan L, Kochi E, et al. mHealth innovations as health system strengthening tools: 12 common applications and a visual framework. Glob Health Sci Pract 2013;1:160-71.

11. Krause HG, Natukunda H, Singasi I, et al. Treatmentseeking behaviour and social status of women with pelvic organ prolapse, 4th degree obstetric tears, and obstetric fistula in western Uganda. Int Urogynecol J 2014;25:1555-9.

12. Crawford J, Larsen-Cooper E, Jezman Z, et al. SMS versus voice messaging to deliver $\mathrm{MNCH}$ communication in rural Malawi: assessment of delivery success and user experience. Glob Health Sci Pract 2014;2:35-46.

13. Bell O. The blind spot of SMS projects: Constituent illiteracy. Available online: https://www.ictworks.org/theblind-spot-of-sms-projects-constituient-illiteracy/

14. Dolea C, AbouZahr C. Global Burden of Obstructed Labour in the Year 2000. World Health Organization, Geneva, 2003.

15. Adler AJ, Ronsmans C, Calvert C, et al. Estimating the prevalence of obstetric fistula: a systematic review and meta-analysis. BMC Pregnancy Childbirth 2013;13:246.

16. Lyimo MA, Mosha IH. Reasons for delay in seeking treatment among women with obstetric fistula in Tanzania: a qualitative study. BMC Womens Health 2019;19:93.

17. Mselle LT, Moland KM, Evjen-Olsen B, et al. "I am nothing": experiences of loss among women suffering from severe birth injuries in Tanzania. BMC Womens Health 2011;11:49.

18. Direct Relief International. Global Fistula Map. Available online: https://www.globalfistulamap.org/

19. Fistula Care Plus. Annual Reports. Available online: https://fistulacare.org/resources/program-reports/

20. Sripad P, Johnson C, Tripathi V, et al. Comparing three models of fistula care among five facilities in Nigeria and Uganda. In: Drew LB, Ruder B, Schwartz DA, editors. A Multidisciplinary Approach to Obstetric Fistula in Africa Public Health, Anthropological and Medical Perspectives. Springer Publishing, New York, In press.

21. Baker Z, Bellows B, Bach R, et al. Barriers to obstetric fistula treatment in low-income countries: a systematic 
review. Trop Med Int Health 2017;22:938-59.

22. Keya KT, Sripad P, Nwala E, et al. "Poverty is the big thing": exploring financial, transportation, and opportunity costs associated with fistula management and repair in Nigeria and Uganda. Int J Equity Health 2018;17:70.

23. Warren C, Agbonkhese R, Ishaku S. Formative Research on Factors Influencing Access to Fistula Care and Treatment in Uganda. Population Council, New York, 2016.

24. Sripad P, Warren C. Formative Research on Factors Influencing Access to Fistula Care and Treatment in Uganda. Population Council, New York, 2016.

25. Khisa W, Wakasiaka S, Kagema F, et al. Contraception knowledge and practice among fistula patients at referral centers in Kenya. Int J Gynaecol Obstet 2012;118:220-2.

26. Michie S, van Stralen MM, West R. The behaviour change wheel: a new method for characterising and designing behaviour change interventions. Implement Sci 2011;6:42.

27. Ayakaka I, Ackerman S, Ggita JM, et al. Identifying barriers to and facilitators of tuberculosis contact investigation in Kampala, Uganda: a behavioral approach. Implement Sci 2017;12:33.

28. Ayton DR, Barker AL, Morello RT, et al. Barriers and enablers to the implementation of the 6-PACK falls prevention program: A pre-implementation study in hospitals participating in a cluster randomised controlled trial. PLoS One 2017;12:e0171932.

29. Tripathi V, Arnoff E, Sripad P. Removing barriers to fistula care: Applying appreciative inquiry to improve access to screening and treatment in Nigeria and Uganda. Health Care Women Int 2019:1-16. [Epub ahead of print].

30. Mallick, L. Comparative Analysis of a Proxy Measurement of Vaginal Fistula: Supplement to DHS Analytical Studies No. 17. ICF International, Rockville, 2016.

31. Uganda Bureau of Statistcs (UBOS) and ICF. Uganda Demographic and Health Survey 2016: Key Indicators Report. UBOS, Kampala, 2017.

32. Maheu-Giroux M, Filippi V, Samadoulougou S, et al. Prevalence of symptoms of vaginal fistula in 19 sub-Saharan Africa countries: a meta-analysis of national household survey data. Lancet Glob Health 2015;3:e271-8.

33. Sripad P, Nwala E, Tripathi V. Reducing barriers to accessing fistula repair: Implementation research in Ebonyi. Population Council, New York, 2018.

34. Sripad P, Nwala E, Tripathi V. Reducing barriers to accessing fistula repair: Implementation research in Katsina. Population Council, New York, 2018.
35. Barageine J, Ndwiga C, Kanakulya H, et al. Reducing barriers to accessing fistula repair: Implementation research in Uganda. Population Council, New York, 2018.

36. Blanc AK, Glazer K, Ofomata-Aderemi U, et al. Myths and Misinformation: An Analysis of Text Messages Sent to a Sexual and Reproductive Health Q\&A Service in Nigeria. Stud Fam Plann 2016;47:39-53.

37. Green EP, Whitcomb A, Kahumbura C, et al. "What is the best method of family planning for me?": a text mining analysis of messages between users and agents of a digital health service in Kenya. Gates Open Res 2019;3:1475.

38. Tunçalp Ö, Isah A, Landry E, et al. Community-based screening for obstetric fistula in Nigeria: a novel approach. BMC Pregnancy Childbirth 2014;14:44.

39. National Bureau of Statistics. 2017 Demographics Statistics Bulletin. NBOS, Abuja, 2018.

40. City Population. Nigeria: Administrative Divisions. Available online: https://www.citypopulation.de/php/ nigeria-admin.php

41. Uganda Bureau of Statistics. The National Population and Housing Census 2014 - Area Specific Profile Series, Kampala, Uganda. UBOS, Kampala, 2017.

42. Forenbacher I, Husnjak S, Cvitić I, et al. Determinants of mobile phone ownership in Nigeria. Telecommunications Policy 2019;43:101812.

43. Burjorjee DM, Bin-Humam Y. New insights on women's mobile phone ownership. Consultative Group to Assist the Poor, Washington, 2018.

44. Ag Ahmed MA, Gagnon MP, Hamelin-Brabant L, et al. A mixed methods systematic review of success factors of mhealth and telehealth for maternal health in Sub-Saharan Africa. Mhealth 2017;3:22.

45. LeFevre AE, Dane P, Copley CJ, et al. Unpacking the performance of a mobile health information messaging program for mothers (MomConnect) in South Africa: evidence on program reach and messaging exposure. BMJ Glob Health 2018;3:e000583.

46. Labrique A, Vasudevan L, Weiss W, et al. Establishing Standards to Evaluate the Impact of Integrating Digital Health into Health Systems. Glob Health Sci Pract 2018;6:S5-17.

doi: $10.21037 /$ mhealth.2019.12.04

Cite this article as: Tripathi V, Arnoff E, Bellows B, Sripad P. Use of interactive voice response technology to address barriers to fistula care in Nigeria and Uganda. mHealth 2020;6:12. 